\title{
Post-exposure prophylaxis for HIV infection after sexual assault: When is it indicated?
}

\author{
C Fong
}

\begin{abstract}
To date there are no published studies on the effectiveness of HIV post-exposure prophylaxis (PEP) after sexual exposure. However, as emergency physicians we are faced with the dilemma of assessing the risk of HIV transmission and providing appropriate care for patients who present to us after sexual assault. Current practice in UK emergency departments and genitourinary clinics varies from trust to trust. There is no direct evidence that HIV $P E P$ after rape prevents transmission or is cost effective. However, there may be certain rape cases where HIV PEP should be prescribed or at least considered. While we wait for more definitive clinical studies, HIV PEP after rape should be considered on a case to case basis. This article aims to provide emergency physicians with current scientific evidence on HIV PEP enabling them to make an informed decision on when HIV PEP is indicated after rape.

(Emerg Med f 2001;18:242-245)
\end{abstract}

Keywords: HIV; post-exposure prophylaxis; sexual assault

Although the risks of HIV transmission and the efficacy of post-exposure prophylaxis (PEP) have been well delineated in occupational settings, there are inadequate data regarding PEP in post-sexual assault cases. There are no current guidelines on PEP for rape victims. The UK Department of Health in their document entitled "Guidelines on Post-Exposure Prophylaxis for Health Care Workers Occupationally exposed to HIV" (June 1997) have stated that the efficacy of PEP after sexual exposure has not been studied and that clinicians will have to consider the circumstances in each case. ${ }^{1}$ Some of the problems faced by researchers in studying the effectiveness of PEP in non-occupational settings include the ethical obstacles to a placebo controlled trial, the need for large sample sizes and follow up of patients. Nevertheless, as clinicians we often have to make decisions under conditions of uncertainty until more definitive evidence becomes available. We have formulated a simple guideline that may help the emergency physician decide when HIV PEP is appropriate after sexual assault. However, as with many guidelines, it may have to be adapted to each physician's practice and available resources.

Risk of source being HIV positive

The first consideration is the HIV seroprevalence of the relevant population. The likelihood of seropositivity is higher in certain population groups. For example if the rape occurred in a male prison, it can be considered that the source is very likely to be HIV positive and the risk of transmission will be fairly high. In the US, the incidence of AIDS is approximately 14 times higher in the prison population (202 cases per 100000 prisoners) than in the general population (14.65 cases per 100000 population). ${ }^{2}$

The number of reported cases and risk groups also differ from country to country and in different subpopulations. By June 1994, 396015 cases had been reported in the USA whereas 10156 cases were reported in the UK by December $1994 .^{3}$ Most of those affected were men ( $92 \%$ in UK, $87 \%$ in US). Apart from unprotected homosexual intercourse, other risk taking behaviours that increase HIV transmission include, intravenous drug misuse and unprotected heterosexual sexual intercourse with intravenous drug misusers or bisexual men. In Europe a further risk group can be identified in the subpopulation of patients from Central Africa where heterosexual intercourse is the main route of transmission with a ratio of infected men: infected women of virtually $1: 1 .^{3}$

\section{Risk of HIV transmission if the assailant} is HIV positive

The risk of occupational HIV transmission after percutaneous or needlestick injury exposure involving body fluid from an HIV infected source is $0.25 \%(9 / 3628 ; 95 \%$ CI $0.12 \%$ to $0.47 \%) .{ }^{4}$ This risk increases if there are large amounts of blood, high titres of HIV or deep injuries involved. Mucous membrane exposure to an HIV infected source carries a risk of $0.09 \%(1 / 1007 ; 95 \%$ CI $0.006 \%$ to $0.5 \%){ }^{45}$ For men who have anal intercourse with men, the risk of HIV transmission after a single act of receptive anal intercourse with a HIV positive partner has been estimated at $0.5-3 \%$ 
Table 1 Estimated likelihood of HIV transmission with different modes of exposure ${ }^{9}$

\begin{tabular}{ll}
\hline Mode of exposure & $\begin{array}{l}\text { Likelihood of transmission } \\
\text { (per 10 000 exposures) }\end{array}$ \\
\hline Receptive anal intercourse & $10-30$ \\
Receptive vaginal intercourse & $8-20$ \\
Needle sharing injection & 67 \\
$\begin{array}{l}\text { Percutaneous (health care } \\
\quad \text { workers) }\end{array}$ & 30 \\
Insertive anal sex & 3 \\
Insertive vaginal sex & $3-9$ \\
\hline
\end{tabular}

using a binomial model based on data collected from 155 homosexual partnerships. ${ }^{6}$ This is a higher risk than that associated with percutaneous exposure. For discordant heterosexual couples, it is estimated that $0.1 \%$ of acts of penile-vagina intercourse lead to HIV transmission, with the risk being higher for male to female transmission. ${ }^{4}$

The risk of HIV sexual transmission is highly variable and depends on the infectiousness of the source partner or in the case of rape, the assailant. These include viral load, virulence of the strain, clinical stage of infection (with the recently infected and those at late stages being most infectious), mucosal damage and concomitant genital tract infections. ${ }^{28}$ The prevalence of genital ulcer disease is associated with an increased relative risk of HIV infection, ranging from 1.5 to 7.0 in both men and women. ${ }^{8}$ Gonorrhoea, chlamydia and trichomonas infection are associated with a relative increase of $60 \%$ to $340 \%$ in the prevalence of HIV infection in men and women. ${ }^{8}$ This association between genital tract infections and increase susceptibility to HIV infection remains high even after adjusting for sexual behaviour. Furthermore, actual measurements of HIV in genital secretions are higher if there is a concomitant sexually transmitted disease (STD).

Table 1 compares the estimated likelihood of HIV transmission during unprotected sex or contaminated needle exposure when the source is HIV positive. ${ }^{9}$ We can see that sexual intercourse (especially receptive anal intercourse) from an HIV positive source carries a small but similar risk of HIV transmission to percutaneous/needlestick injuries in health care workers. HIV PEP is routinely offered to health workers exposed to HIV percutaneously. Should rape patients exposed to a HIV positive source be offered HIV PEP? The main problem here is that the HIV status of the assailant in sexual assault is often unavailable whereas in the occupational setting the HIV status of the source patient is either known or can usually be readily determined. Thus, the clinician should consider the probability of the source being HIV positive and whether the route of transmission was high risk as part of the decision analysis.

\section{Factors increasing the risk of HIV transmission}

We must also consider the victim's susceptibility to infection. There is an increased risk of transmission after rape as compared with consensual sex as a result of genital or rectal trauma leading to bleeding. ${ }^{2}$ Furthermore, there may be multiple assailants or exposure to multiple receptive sites including scratches, abrasions and lacerations that may involve percutaneous transmission. Rape may also increase the risk of HIV transmission because sexually transmitted diseases are common after rape. Up to $20 \%$ of all penetrative rapes of women by men result in trichomoniasis or bacterial vaginosis and up to $5 \%$ result in chlamydia or gonorrhea. ${ }^{9}$ These infections may increase the susceptibility to HIV infection as discussed earlier.

\section{Efficacy and safety of PEP}

The use of zidovudine (AZT) after occupational exposure to HIV was associated with a $79 \%$ reduction in the odds of HIV transmission $(\mathrm{OR}=0.21 ; 95 \% \mathrm{CI} 0.10,0.60)$ in the Centers of Disease Control and Prevention (CDC) study. ${ }^{10}$ Further evidence to support the use of PEP comes from the use of AZT in prevention of perinatal transmission. AZT given to pregnant women orally starting at between 14 and 34 weeks, then intravenously during delivery and orally to the neonate for six weeks reduced vertical transmission rates at age 18 months from $25.5 \%$ to $8 \%$ when compared with placebo. ${ }^{11}$ Recent placebo controlled trials showed that short courses of AZT (given orally to the mother; starting at between 36-38 weeks of pregnancy, continuing during delivery, and for a week after delivery) reduced the risk of transmission of HIV to the baby at 3-6 months by about $37-50 \% .{ }^{11}$ Current UK guidelines now recommend starting AZT in the third trimester to prevent vertical transmission. ${ }^{12}$

The efficacy of HIV PEP after consensual and non-consensual sex is unknown. Studies are currently being carried out in the United States to determine whether the regimen used for health care workers is effective in nonoccupational exposures. ${ }^{13}$ The six month multicentre HIV Network for Prevention trials (HIVNET) trial will involve homosexual men who are at high risk for HIV exposure randomised to PEP or "usual care" after a recent exposure. However, these studies are only investigating how access to prophylaxis will affect behaviour and whether people at risk for HIV can maintain the multidrug regimen. The San Francisco Department of Public Health is also conducting a study to test the feasibility of providing PEP to men and women exposed to HIV through sexual contact or IV drug use. ${ }^{13}$

\section{Available regimens}

The current choice of PEP drugs after a high risk occupational exposure - that is, body fluids or tissues known to be, or strongly suspected to be, infected with HIV through percutaneous exposure, mucous membrane exposure or through broken skin recommended by the UK health department ${ }^{1}$ are:

Zidovudine (AZT) $200 \mathrm{mg}$ thrice daily or 250 mg twice daily plus

Lamivudine $150 \mathrm{mg}$ twice daily plus

Indinavir $800 \mathrm{mg}$ thrice daily 
Taken for four weeks

PEP is most effective within two hours of exposure, but is recommended up to 72 hours after exposure.

In 1998 the Centers for Disease Control ${ }^{9}$ recommended zidovudine and lamivudine for PEP in sexual assault cases. They also recommended that a protease inhibitor such as indinavir should be added to the regimen if the assailant is known to be infected with HIV resistant to reverse transcriptase inhibitors. Therefore, unless the assailant is known to be HIV positive, it is reasonable to offer zidovudine and lamivudine to the sexual assault patient for four weeks. The addition of indinavir increases the risk of side effects, such as renal stones and decreases compliance as it has to be taken on an empty stomach. ${ }^{12}$ Other common side effects of zidovudine and lamivudine include nausea and gastrointestinal symptoms, myalgia, anaemia and neutropenia. Zidovudine is the only drug for which extensive safety data during pregnancy are available and it seems to be safe even in the first trimester. ${ }^{12}$

\section{Practical management of the victim}

Despite the lack of data on PEP in sexual exposure/assault, PEP should be offered to the sexual assault victim once the clinician has assessed all the factors involved in the likelihood of HIV transmission. PEP should be viewed in the overall context of the physical and psychological trauma of the rape victim; PEP might help the victim gain a sense of control and decrease their anxiety about acquiring HIV. The patient should be reassured that the risk of transmission is small, may increase with certain risk factors, and PEP may or may not

Sexual assault with evidence of penetration

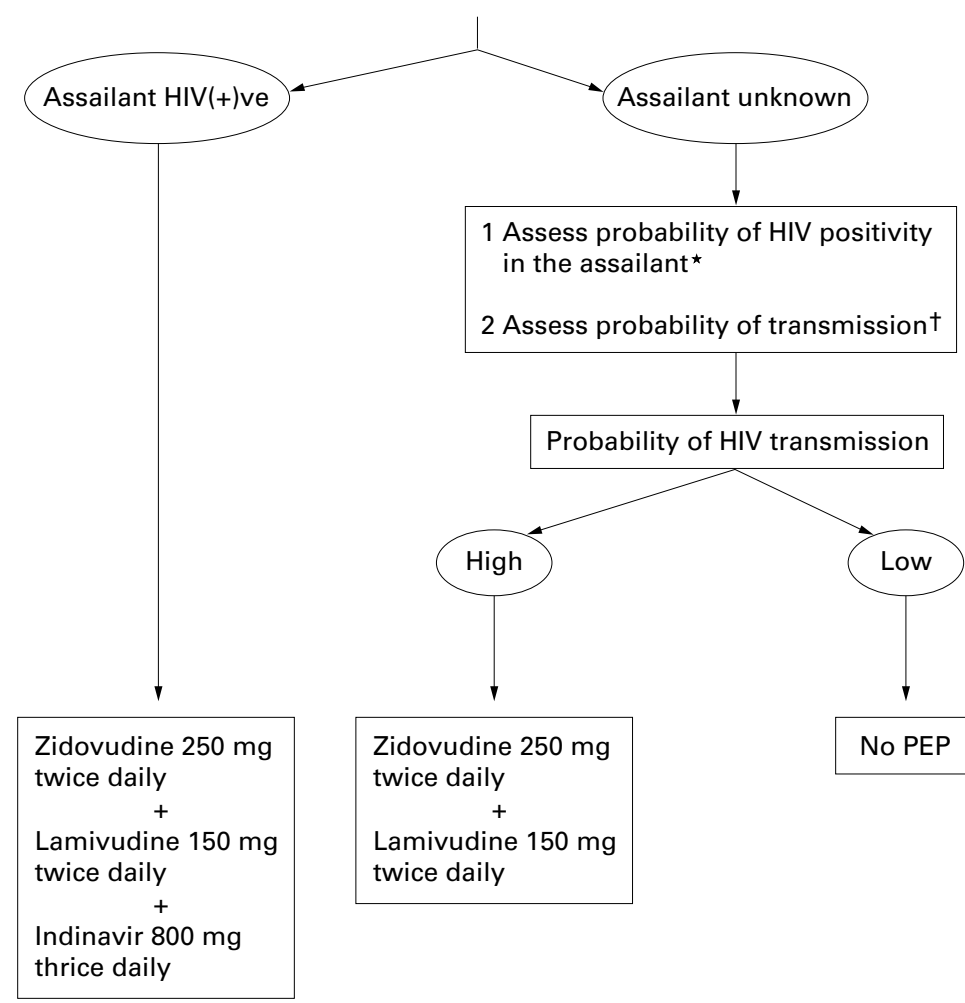

Figure 1 A suggested guideline for HIV PEP after sexual assault. be indicated in their case. The patient should be informed of its questionable efficacy and that until further studies are carried out, our knowledge of PEP is largely based on PEP in occupational exposure.

The management and care of the sexual assault victim should include medical evaluation and treatment, forensic analyses, emotional and mental health support and legal support. HIV counselling and PEP is but one aspect of the care and treatment of the rape victim. Baseline HIV antibody testing should be performed during the initial visit, with conventional assays. Most infected people will have detectable antibodies four to six weeks after exposure and almost all will have seroconverted by six months. ${ }^{14}$ The clinician should explain the "window" period to the patient emphasising they may not seroconvert until six months, thus, they cannot be sure that they are uninfected until a second antibody test is taken six months after exposure. ${ }^{9}$ Some centres would argue that repeat HIV antibody test at six months after exposure would be unnecessary as the newer HIV antibody testing are sensitive enough to pick up early seroconversion. In the meantime, conservative advice is to avoid sexual activity that may transmit HIV. Follow up counselling is imperative as the patient may not be receptive immediately after the assault. This issue is a particularly sensitive one, as the victim is very likely to acquiesce to whatever recommendation the physician makes. Informed consent in this situation has little meaning, so the decision to raise the topic and to offer advice must be made with this in mind.

\section{Public health and ethical issues of PEP}

It is important to emphasise to the public that PEP will only be offered to patients on a case by case basis and even then has its side effects and may not be appropriate for all exposures. Even in health workers (who can be considered a well motivated and well informed group), at least one third of those who were on PEP stopped treatment because of intolerance to the side effects. ${ }^{9}$ So poor adherence to treatment may be a problem leading to prophylaxis failure. The risk of poor compliance should not deter the clinician from offering PEP to sexual assault victims. Instead strategies for improving compliance such as offering anti-emetics, educating the patients, providing a 24 hour hot line and liaison with local GPs may help to prevent poor adherence.

Health policy makers will no doubt question the cost effectiveness of PEP in sexual assault. Currently the costs of a four week course of zidovudine $=£ 174$, lamivudine $=£ 170$ and indanivir $=£ 224$. There are further costs involved in HIV testing, monitoring and treating the side effects of the drugs and further unquantified costs involved in counselling and follow up consultations by HIV health care teams. The cost effectiveness of treatment is still not known but will depend on the likelihood that the source patient is HIV positive, the risk of transmission, and the effectiveness of PEP. These are the same three factors on which we have based our guideline. 
The true cost effectiveness of PEP will not be evident until some of the feasibility studies currently underway in the United States are completed. Cost-benefit analysis comparing the costs of preventing HIV and the costs of treating HIV after sexual assault could be made subsequent to these studies.

\section{A suggested guideline for HIV PEP after sexual assault}

The steps shown in figure 1 are meant to guide the clinician in deciding whether PEP is appropriate after sexual assault on an individual basis.

All patients should receive HIV antibody testing, counselling on the risk of transmission, counselling on the efficacy and risks of PEP, and counselling on prevention and safe sex (fig 1).

$1{ }^{\star}$ Probability of $\mathrm{HIV}(+)$ in the assailant. A high risk profile includes:

- IV drug user

- Practices unsafe sex

- Bisexual / homosexuality

- Promiscuity

- Comes from a high HIV seroprevalence population

If known to be HIV (+)ve need to consider:

- Clinical stage of HIV infection

- Quantitative HIV load

- History of retroviral treatment

$2{ }^{\dagger}$ Factors increasing risk of transmission

- Presence of concomitant STD

- Presence of genital trauma and bleeding

- Presence of inflammation

- Presence of extragenital trauma and lacerations

- Multiple exposure including multiple assailants

3 Factors affecting the effectiveness of treatment

- Time from exposure

(Maximum effectiveness within two hours, ineffective beyond 72 hours)

- Likelihood of compliance with treatment

4 Special circumstances

- Children/adolescents

- Pregnant women

- Source of HIV known to have antiretroviral resistant viruses

(these cases should be discussed with paediatricians and infectious diseases physicians for treatment and follow up)

When PEP is offered after sexual assault the following tests are indicated:

- HIV antibody testing (repeated at six weeks, three months and six months)

- Hepatitis B virus antibody test
- Gonorrhea, chlamydia and syphilis test

- Wet mount for trichomonas

- Pregnancy test in women of childbearing age

- Liver function tests

- Full blood count

\section{Summary}

The management and care of the rape patient is complicated and requires medical, psychological, social and legal considerations. We have only focused on one aspect of this subject, HIV PEP, which remains controversial because of a lack of clinical trials. Sexual assault itself is often underreported and underestimated and this makes the study of HIV transmission after sexual assault difficult. The risk of HIV transmission after sexual assault is multifactorial and although small may be significant in certain circumstances. It is therefore not unreasonable for patients at risk of HIV infection presenting to the emergency department after sexual assault to be offered HIV PEP. It is hoped that this discussion of the current available scientific knowledge and simple guideline will enable the clinician to make an informed decision on when to offer PEP to patients after sexual assault.

The author would like to thank Professor John Henry and $\mathrm{Mr}$ Robin Touquet for their encouragement and invaluable advice in preparing this article. Special thanks to Dr Paul Halley for proof reading the article.

1 UK Health Department. Guidelines on post-exposure prophylaxis for health care workers occupationally exposed to HIV. London: UK Health Department, June 1997.

2 Gostin L, Lazzarini Z, Alexander D, et al. HIV testing, counseling and prophylaxis after sexual assault. $\mathscr{F} A M A$ 1994; 271:1436-44

3 Adler M, Weller I, Gilson R, et al. ABC of sexually transmitted diseases. 3rd edition. London: BMJ Publishing Group, 1995:33.

4 Lurie P, Miller S, Hecht F, et al. Postexposure porphylaxis after nonoccupational HIV exposure. FAMA 1998;280: 1769-73.

5 Ippolito G, Puro V, De Carli G and the Italian Study Group on Occupational Risk of HIV Infection. The risk of occupational human immunodeficiency virus infection in health care workers. Italian multicentre study. Arch Intern Med 1993;153:1451-8.

6 DeGruttola V, Seage III G, Mayer K, et al. Infectiousness of DeGruttola V, Seage III G, Mayer K, et al. Infectiousness of
HIV between male homosexual partners. F Clin Epidemiol

7 Mastro TD, deVincenzi I. Probabilities of sexual HIV-1 transmission. AIDS 1996;10 (suppl A):S75-8.

8 Royce R, Sena A, Cates W Jr, et al. Sexual transmission of HIV. N Engl f Med 1997;336:1072-8.

9 Bamberger JD, Waldo CR, Gerberding JL, et al. Postexposure prophylaxis for human immunodeficiency virus (HIV) infection following sexual assault. Am f Med 1999;106: 323-6.

10 Gerberding L. Prophylaxis for occupational exposure to HIV. Ann Intern Med 1996;125: 497-501.

11 Connor EM, Sperling RS, Gelber R and the Pediatric AIDS Clinical Trials Group Protocol 076 Study Group. Reduction of maternal-infant transmission of human immunotion of maternal-infant transmission of human immunoMed 1994;331:1173-80.

12 Collier J. HIV in pregnancy and early childhood. Drug Ther Bull 1999;37:65-7.

13 Vachiere C. Is HIV prophylaxis effective for sexual Vachiere C. Is HIV prophylaxis effective
exposures? Ann Intern Med 1998;128:513-14.

14 Katz M, Gerberding JL. The care of persons with recent sexual exposure to HIV. Ann Intern Med 1998;128:306-12. 